\title{
Sarcopenic obesity in liver cancer: it is SO complicated
}

\author{
Gyorgy Baffy \\ Department of Medicine, VA Boston Healthcare System and Brigham and Women's Hospital, Harvard Medical School, Boston, MA, USA \\ Correspondence to: Gyorgy Baffy, MD, PhD. VA Boston Healthcare System, 150 South Huntington Avenue, Room 6A-46, Boston, MA 02130, USA. \\ Email: gbaffy@bwh.harvard.edu. \\ Comment on: Kobayashi A, Kaido T, Hamaguchi Y, et al. Impact of Sarcopenic Obesity on Outcomes in Patients Undergoing Hepatectomy for \\ Hepatocellular Carcinoma. Ann Surg 2019;269:924-31.
}

Submitted Jul 15, 2019. Accepted for publication Jul 25, 2019.

doi: 10.21037/hbsn.2019.07.16

View this article at: http://dx.doi.org/10.21037/hbsn.2019.07.16

Sarcopenia is defined as a generalized and progressive loss of skeletal muscle mass, strength and function (1). Sarcopenia, while initially described in the aging general population, affects many patients with various chronic diseases in all age groups. Diminished muscle volume and performance is associated with lower endurance, frailty, disability, and poor disease outcomes. To detect sarcopenia, muscle mass can be determined by bioelectrical impedance analysis (BIA), dual energy X-ray absorptiometry or by computed tomographyguided calculations such as the psoas muscle area or skeletal muscle index (SMI) derived from lumbar third vertebral (L3) cross-sectional measurements, while muscle strength and function is mainly assessed by clinical exams such as grip strength, 6-minute walking distance and the get-up-and-go test. However, the diagnostic criteria of sarcopenia have not been firmly established and clinical research and practice often depend on morphological estimates alone (1).

Obesity as a highly prevalent condition increasingly coincides with sarcopenia. Sarcopenic obesity (SO) has been introduced as a term for the simultaneous presence of skeletal muscle loss and excess body fat, although sarcopenia and obesity remain defined by separate criteria and it is debated whether the impact of SO on clinical outcomes is more than the sum of its components (2). Of note, both components of SO have been associated with insulin resistance and chronic systemic low-grade inflammation, suggesting overlapping molecular pathways and shared targets in their pathophysiology. Myosteatosis (ectopic fat accumulation in skeletal muscle) is a characteristic feature of SO that can be diagnosed by muscle biopsy, computed tomography or magnetic resonance spectroscopy. Myosteatosis is associated with impaired muscle function

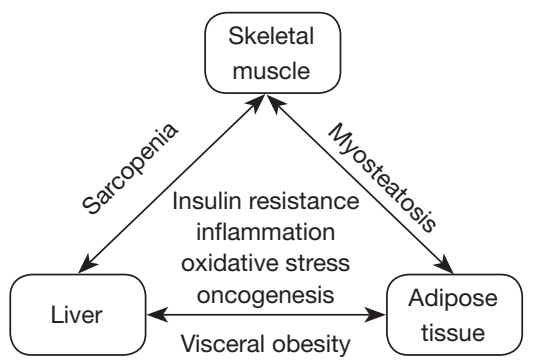

Figure 1 Adipose-muscle-liver axis and clinical components of sarcopenic obesity. Key pathophenotypes contributing to poor outcomes in chronic liver disease and major complications such as hepatocellular carcinoma are shown.

and it may also affect individuals who do not meet anthropometric criteria for either sarcopenia or obesity. Myosteatosis impairs glucose uptake in skeletal muscle and contributes to insulin resistance, while diminished glycogen pools in the cirrhotic liver promote 'accelerated starvation' and muscle breakdown (3). These and many other interactions within the adipose tissue-skeletal muscle-liver triangle may explain why SO represents a major concern in advanced liver disease (Figure 1). Indeed, the prevalence of sarcopenia is particularly high among patients with cirrhosis, conferring lower survival rates and increased risk of complications such as infections. Moreover, sarcopenia is associated with the development of hepatocellular carcinoma (HCC), predicts tumor recurrence, and doubles the risk of all-cause mortality (4).

To determine the specific role of SO in the prognosis of HCC, a retrospective analysis by Kobayashi et al. (5) 
compared the outcomes of primary hepatectomy for HCC in 465 patients, treated at Kyoto University in Japan and grouped by the presence or absence of sarcopenia and obesity. Sarcopenia was defined by SMI calculated from L3 level cross-sectional areas of skeletal muscle normalized for body height with a gender-specific cutoff and obesity was defined in both genders by the visceral fat area (VFA). Based on these parameters, $13.4 \%$ of patients had sarcopenia and $53.8 \%$ of patients had obesity, while $6.7 \%$ had overlapping conditions defined as SO. Overall survival and recurrencefree survival rates were significantly lower in sarcopenic patients when compared to non-sarcopenic patients with or without the presence of obesity. Notably, BMI definition of obesity would have classified half as many patients into the obese group (25.2\%). Authors concluded that obesity was not a negative prognostic factor unless it occurred in combination with sarcopenia, providing no support for the role of $\mathrm{SO}$ as a separate entity in postoperative outcomes of HCC (5).

In a parallel work, the Kyoto group reported another analysis on the clinical outcomes of 606 patients with primary or secondary hepatectomy for HCC in the presence or absence of sarcopenia and obesity (6). In this work, sarcopenia was assessed by SMI, while the authors also calculated intramuscular adipose tissue content (IMAC) to determine myosteatosis. Moreover, obesity was assessed by the visceral-to-subcutaneous adipose tissue area ratio (VSR) rather than by VFA. This is a more complex criterion for obesity since VSR accounts for the potentially beneficial impact of subcutaneous adipose tissue that may lie behind controversial observations on the 'obesity paradox'. Reanalysis of the (mostly overlapping) Kyoto cohort according to the presence of visceral obesity (VSR), sarcopenia (SMI) and myosteatosis (IMAC) found that each of these 3 factors independently predicted mortality after hepatectomy for HCC. These data support therefore an additive effect between sarcopenia and obesity on poor outcomes of HCC and confirm prior observations on another large Japanese cohort of patients with various stages of HCC (7).

Thus, while SO is not necessarily a sovereign pathophysiological entity, there is now evidence that coincidence of two unfavorable conditions may confer worse clinical outcomes in HCC. However, most analyses on how sarcopenia and SO affect HCC so far have been performed on Asian patient cohorts and the findings cannot be readily extrapolated to other geographic regions where anthropometric cutoffs as well as the etiology and prevalence of HCC are different. Yet, as nonalcoholic fatty liver disease (NAFLD) becomes the leading cause of HCC in many geographical areas, it is difficult to overestimate the global role of SO in disease outcomes. Analysis of Western cohorts now and into the future may further emphasize the significance of $\mathrm{SO}$ in the progression of chronic liver disease and its complications.

$\mathrm{SO}$ is a complex nutritional problem that offers a number of areas for future liver research. Beyond the obviously unmet need of having standardized criteria for its definition and identifying effective tools for its management, only a few areas can be named here. First, interventional protocols need to be optimized to reduce the risk of postoperative sarcopenia that may persist or even newly develop in cirrhotic patients who undergo hepatectomy or liver transplantation for HCC. Second, while improving preinterventional muscle mass and function has great benefits, it remains to be seen how much time can we safely spend to achieve this goal for patients with HCC through nutrition, resistance training and pharmacological means. Finally, we may improve risk prediction by routinely including muscle quality and strength in the assessment of sarcopenia. Myosteatosis, which is increasingly recognized as an adverse manifestation of obesity and the metabolic syndrome, appears to have a distinct pathogenic role and could become an important risk assessment tool in liver disease.

\section{Acknowledgments}

None.

\section{Footnote}

Conflicts of Interest: The author has no conflicts of interest to declare.

Ethical Statement: The author is accountable for all aspects of the work in ensuring that questions related to the accuracy or integrity of any part of the work are appropriately investigated and resolved.

\section{References}

1. Cruz-Jentoft AJ, Sayer AA. Sarcopenia. Lancet 2019;393:2636-46.

2. Polyzos SA, Margioris AN. Sarcopenic obesity. Hormones (Athens) 2018;17:321-31.

3. Eslamparast T, Montano-Loza AJ, Raman M, et al. Sarcopenic obesity in cirrhosis-The confluence of 2 
prognostic titans. Liver Int 2018;38:1706-17.

4. Chang KV, Chen JD, Wu WT, et al. Association between Loss of Skeletal Muscle Mass and Mortality and Tumor Recurrence in Hepatocellular Carcinoma: A Systematic Review and Meta-Analysis. Liver Cancer 2018;7:90-103.

5. Kobayashi A, Kaido T, Hamaguchi Y, et al. Impact of Sarcopenic Obesity on Outcomes in Patients Undergoing Hepatectomy for Hepatocellular Carcinoma. Ann Surg 2019;269:924-31.

Cite this article as: Baffy G. Sarcopenic obesity in liver cancer: it is sarcopenic obesity complicated. HepatoBiliary Surg Nutr 2019;8(5):560-562. doi: 10.21037/hbsn.2019.07.16
6. Hamaguchi Y, Kaido T, Okumura S, et al. Preoperative Visceral Adiposity and Muscularity Predict Poor Outcomes after Hepatectomy for Hepatocellular Carcinoma. Liver Cancer 2019;8:92-109.

7. Fujiwara N, Nakagawa H, Kudo Y, et al. Sarcopenia, intramuscular fat deposition, and visceral adiposity independently predict the outcomes of hepatocellular carcinoma. J Hepatol 2015;63:131-40. 